\title{
Prevention and Treatment of Basic Diseases: A Potential Direction to Intervene Alzheimer's Disease
}

\author{
Wei Jiangping ${ }^{1,2}$ and Xu Shijun ${ }^{1,2^{*}}$ \\ ${ }^{1}$ School of Pharmacy, Chengdu University of Traditional Chinese Medicine, Chengdu, China \\ ${ }^{2}$ Institute of Meterial Medica Integration and Transformation for Brain Disorders, CDUTCM, China
}

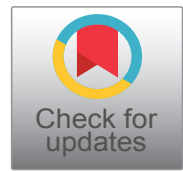

*Corresponding author: Shijun $\mathrm{Xu}, \mathrm{PhD}$, School of Pharmacy, Chengdu University of Traditional Chinese Medicine, Chengdu, 611137, China, Tel: +8613111898478

\begin{abstract}
As the life span of human beings increases, the number of dementia patients such as Alzheimer's disease (AD) is increasing. $A D$ may be one of the major diseases faced by the whole world in the future. It is an unresponsive for single drug according to the current stage of $A D$ pathogenesis and drug research. Combined use of various drugs may be the inevitable trend of $A D$ drug research in the future. Personalized treatment of metabolic diseases, vascular diseases and chronic inflammatory risk diseases is a necessary step to intervene in $A D$. During the middle age, to strengthen immunity and to reduce the external environment to stimulate the susceptible gene, which is beneficial to suppress the formation of $A D$. In a word, the early prevention of $A D$ is better than the later treatment.
\end{abstract}

\section{Keywords}

Alzheimer's disease, Metabolic disease, Cardiovascular disease, Chronic inflammatory disease

Alzheimer's disease (AD) is the most important neurodegenerative disease in the world. At present, there are about 49 million patients worldwide, whose incidence and prevalence are increasing year by year. Since the pathogenesis of $A D$ is not clear, there is no other new drugs were approved for sale in the 15 years after FDA approved memantine in 2003. Many clinical trials of new drugs worldwide ended in failure. In May 17, 2018, Johnson announced the termination of atabecestat development. Pfizer, one of the largest pharmaceutical company in the world, announced to close all new drugs for Alzheimer's disease last year. More brutally, the French Ministry of Health recently confirmed that $A D$ 's existing four drugs did not achieve satisfactory medical results. The lack of clinical demand and new drug listing has been known as the biggest contradiction in the field. It is foreseeable that AD will be one of the major problems of geriatrics and socioeconomic problems facing the world in the future.

\section{Aging is the Precondition for the Formation of $A D$}

It is worth noting that dementia such as $A D$ is not the exclusive disease of the modern people. The Chinese people had the corresponding literature more than 2000 years ago. Huang Di Nei Jing records that women aging begins at 35-years-old, while men aging begins at 40-years-old. This record is much more consistent with the incidence of $A D$ in modern women than in men [1]. Moreover, studies have found that some people aged only more than 30 years can be observed higher level of $\beta$-amyloid in the brain [2]. While Individuals with Down's syndrome have been diagnosed with $A D$ at the age of 30 to 40 [3]. Although these evidences do not prove that $A D$ will be produced after aging, there is clear evidence that the prevalence of $A \beta$ related to age [4]. More importantly, aging is closely related to skin barrier and organ function decline [5-8]. These changes reduce the body's resistance to external stimuli and may trigger immune responses and metabolic abnormalities. And then they induce the risk diseases of AD. Therefore, aging is the most critical risk factor for late-onset $A D$ $[9,10]$.

\section{Basic Disease is the Necessary Condition for the Development of AD}

$A D$ is a complex neurodegenerative disease with many diseases involved in its formation. Type 2 diabetes 
mellitus (T2DM) has been recognized as an important risk disease for $A D$ for many years [11]. Insulin and leptin signal all involve in T2DM. At the same time, they are involved in regulating the functions of nerve and synapse, and the production and clearance of $A \beta$ [11]. Thus, $A D$ and T2DM have the same cellular and molecular mechanism [12]. According to this, AD has also been known as type 3 diabetes mellitus [13]. A recent study found that triglyceride can induce leptin and insulin resistance through going across blood brain barrier (BBB) [14]. TG can inhibit leptin transport across $B B B$ in a dose-dependent manner, while glucose and insulin promote leptin transport [15]. It suggests that these metabolic diseases such as obesity, T2DM and hyperlipidemia are major risk factors for AD. Studies have also shown that obesity induced by high fat diet can inhibit the metabolic activity of the BBB microvessels [16]. And then it causes macrophage invasion and cognitive decline [17]. These evidences suggest that metabolic diseases are one of the most important basal diseases of AD.

Neuroinflammation is an important potential factor in the pathogenesis and development of $A D$ [18]. However, the role of neuroinflammation in the pathological process of $A D$ is not clear. It may be caused by inflammatory factors intruded into the brain through aneretic BBB induced by persistent peripheral disease. It may also be aroused by insufficient local energy supply in the brain. Studies show that $20 \%$ of AD patients have allergic diseases such as allergic rhinitis, bronchial asthma [19]. And appropriate antiasthmatic treatment can improve cognitive ability in AD patients [20]. Unfortunately, there are no reports of allergic diseases and $B B B$ damage. In addition, many studies have shown that rheumatoid arthritis (RA) are related to $A D$ [2124]. The gene and epigenetic regulation of inflammatory cascade is the point of association between $A D$ and RA [24]. More importantly, recent studies have shown that RA patients have BBB dysfunction [25]. And the in- flammatory factor TNF-alpha is a regulator of peripheral inflammation to amyloid pathology [26]. In conclusion, persistent peripheral inflammatory disease may be a major risk factor for neuro-inflammation.

The brain is an organ with high oxygen and energy consumption. The energy supply in the brain is directly related to the normal functioning of brain. In recent years, insufficient supply of AD brain has attracted wide attention [27-30]. In the decades before the symptoms of cognitive decline, AD patients showed chronic cerebral hypoperfusion and glucose hypometabolism [29]. A large number of studies have shown that vascular diseases such as atherosclerosis are the key risk factors for cerebrovascular disorders [31-32]. These cardiovascular diseases are also closely related to peripheral inflammation [33-35]. Thus, they are also one of the risk factors for BBB homeostasis and the development of AD.

\section{Prevention and Control of Basic Diseases is the Fundamental Way to Prevent AD}

Alzheimer's disease is a compound disease caused by many factors. Particulate matter [36,37], herpes virus [38], toxic substances and chemicals [39] are the external factors associated with $A D$ formation. Second, patients may suffer from basic disease due to family inheritance, personal diet, living habits or environmental factors. They can cause a vicious cycle of oxidative stress, inflammation and energy metabolism, which can disturb BBB and even induce the activation of $A D$ susceptible genes. A series of peripheral lesions will gradually form the same vicious cycle in the brain and accelerate the formation of AD typical pathology (Figure 1). Therefore, the protection of BBB is an important direction for the study of neurodegenerative diseases [40].

Aging is a prerequisite for the formation of $A D$, which runs through the whole process of $A D$ lesions. On the premise of aging, the external environment invaded the body, leading to chronic inflammatory dis-

\section{External environment \\ Periphery BBB \\ CNS}

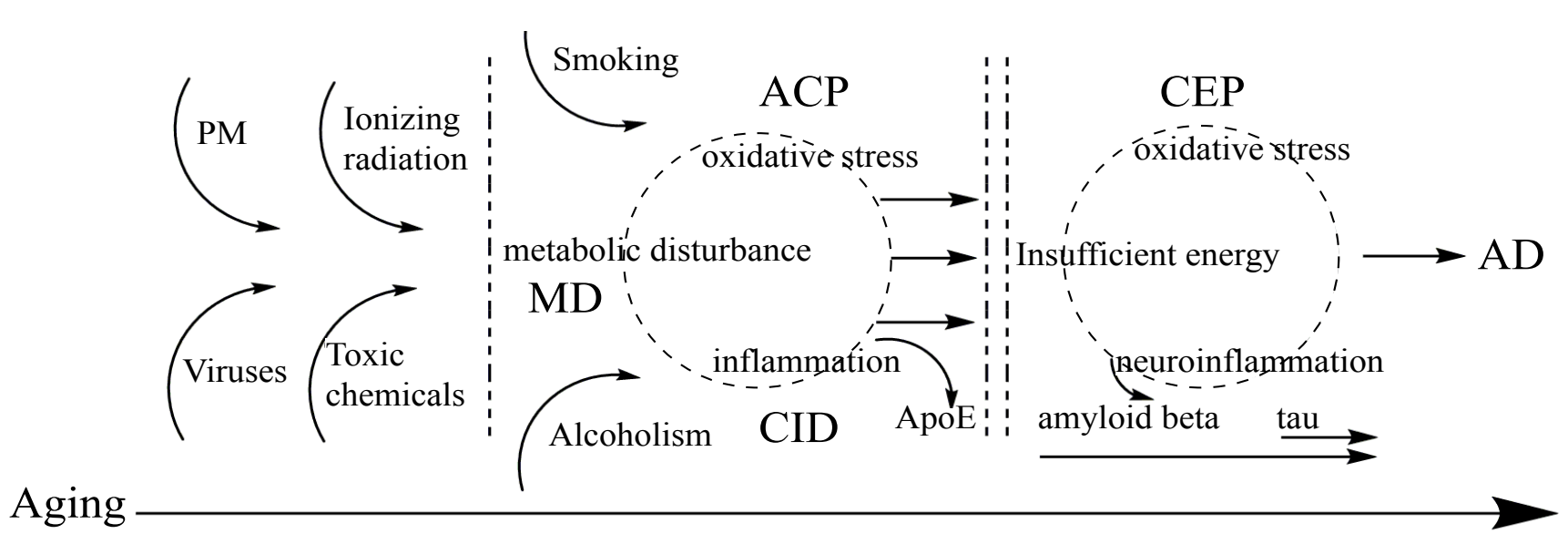

Figure 1: The evolution process of Alzheimer's disease. 
eases (CID). At the same time, angiocardiopathy (ACP) and metabolic diseases (MD) are gradually formed due to personal habits and so on. These basic diseases form a vicious cycle of oxidative stress, inflammation and metabolic disorders. They then invaded the brain by disrupting BBB and induced a similar vicious cycle in the central nervous system (CNS). Subsequently, chronic encephalopathy (CEP) and AD characteristic lesions were induced, and finally $A D$ was formed.

Although the research stage of $A D$ has been moving forward in recent years, it has never been able to get rid of the influence of $A \beta$. At the same time, not all dementia has typical $A \beta$ like lesions, and vice versa. Therefore, it is very important to put $A D$ prevention research on basic diseases. On the one hand, their diagnosis compared with subjective cognitive decline is clearly. On the other hand, when treating basic diseases, we can intervene some external risk factors of patients and avoid the formation of $A D$ as far as possible.

\section{Multi Target Drugs or Combination of Multiple Drugs is a Potential Direction for AD Interven- tion}

Clinically, AD patients are usually accompanied by a variety of basic diseases or other diseases. These diseases are cross-linked, so the failure of many effective laboratory drugs in clinical trials may come from here. Therefore, the therapeutic effect of single target drugs on $A D$ is limited, and the existing treatment drugs are well proven it.

At present, the use of various natural medicines and ethnomedicinal medicine has attracted wide attention $[41,42]$. As the first country to record dementia, there are a large number of prescriptions have been handed down to the present in China. As we all know, Chinese medicine prescription is a combination of various natural medicines according to the rules of Chinese medicine prescription. In addition to interfering with peripheral risk factors, most of the prescriptions for treating $A D$ consider intervening aging (Qi). Unfortunately! These prescriptions are not widely used in clinical since the different principles of treatment. Only part of the research results on animal models are published [43-47]. Anyway, the traditional Chinese medicine prescription may be a potential direction for treating $A D$ in the future.

To sum up, prevention first, treatment second is the basic law to intervene AD. The treatment of basic diseases and the elimination of $A D$ risk factors are the key steps in the treatment of AD. Multitarget drugs or combination of multiple drugs are potential directions for the treatment of AD.

\section{References}

1. Alzheimer's Association (2018) 2018 Alzheimer's Disease Facts and Figures. Alzheimers Dement 14: 367-429.

2. Brookmeyer R, Abdalla N, Kawas CH, Corrada MM (2018) Forecasting the prevalence of preclinical and clinical
Alzheimer's disease in the United States. Alzheimers Dement 14: 121-129.

3. Palat P, Hickey F, Patel L, Sannar E (2018) LevodopaResponsive Early-Onset Parkinsonism in Down Syndrome. Case Rep Neurol Med 2018: 2314791.

4. Roberts RO, Aakre JA, Kremers WK, Vassilaki M, Knopman DS, et al. (2018) Prevalence and Outcomes of Amyloid Positivity Among Persons Without Dementia in a Longitudinal, Population-Based Setting. JAMA Neurol 75: 970-979.

5. Burke KE (2018) Mechanisms of aging and development-A new understanding of environmental damage to the skin and prevention with topical antioxidants. Mech Ageing Dev 172: $123-130$.

6. Sicard D, Haak AJ, Choi KM, Craig AR, Fredenburgh LE, et al. (2018) Aging and anatomical variations in lung tissue stiffness. Am J Physiol Lung Cell Mol Physiol 314: 946-955.

7. Nitta K, Okada K, Yanai M, Takahashi S (2013) Aging and chronic kidney disease. Kidney Blood Press Res 38: 109120.

8. Kwon YH, Kim N, Nam RH, Park JH, Lee SM, et al. (2017) Change in the Interstitial Cells of Cajal and nNOS Positive Neuronal Cells with Aging in the Stomach of F344 Rats. PLoS One 12: e0169113.

9. Hebert LE, Bienias JL, Aggarwal NT, Wilson RS, Bennett DA, et al. (2010) Change in risk of Alzheimer disease over time. Neurology 75: 786-791.

10. Hebert LE, Weuve J, Scherr PA, Evans DA (2013) Alzheimer disease in the United States (2010-2050) estimated using the 2010 Census. Neurology 80: 1778-1783.

11. Paul KC, Jerrett M, Ritz B (2018) Type 2 Diabetes Mellitus and Alzheimer's Disease: Overlapping Biologic Mechanisms and Environmental Risk Factors. Curr Environ Health Rep 5: 44-58.

12. Karki R, Kodamullil AT, Hofmann-Apitius M (2017) Comorbidity Analysis between Alzheimer's Disease and Type 2 Diabetes Mellitus (T2DM) Based on Shared Pathways and the Role of T2DM Drugs. J Alzheimers Dis 60: 721-731.

13. Ahmed S, Mahmood Z, Zahid S (2015) Linking insulin with Alzheimer's disease: emergence as type III diabetes. Neurol Sci 36: 1763-1769.

14. WA Banks, SA Farr, TS Salameh, ML Niehoff, EM Rhea, et al. (2018) Triglycerides cross the blood-brain barrier and induce central leptin and insulin receptor resistance. Int $J$ Obes (Lond) 42: 391-397.

15. Rhea EM, Salameh TS, Logsdon AF, Hanson AJ, Erickson MA, et al. (2017) Blood-Brain Barriers in Obesity. AAPS J 19: 921-930.

16. Suidong Ouyang, Hung Hsuchou, Abba J Kastin, Yuping Wang, Chuanhui Yu, et al. (2014) Diet-induced obesity suppresses expression of many proteins at the blood-brain barrier. J Cereb Blood Flow Metab 34: 43-51.

17. Stranahan AM, Hao S, Dey A, Yu X, Baban B (2016) Bloodbrain barrier breakdown promotes macrophage infiltration and cognitive impairment in leptin receptor-deficient mice. J Cereb Blood Flow Metab 36: 2108-2121.

18. Swanson A, Wolf T, Sitzmann A, Willette AA (2018) Neuroinflammation in Alzheimer's disease: Pleiotropic roles for cytokines and neuronal pentraxins. Behav Brain Res 347: 49-56. 
19. Bożek A, Bednarski P, Jarzab J (2016) Allergic rhinitis, bronchial asthma and other allergies in patients with Alzheimer's disease: unnoticed issue. Postepy Dermatol Alergol 33: 353-358.

20. Bozek A, Jarzab J (2011) Improved activity and mental function related to proper antiasthmatic treatment in elderly patients with Alzheimer's disease. Allergy Asthma Proc 32: 341-345.

21. Chou RC, Kane M, Ghimire S, Gautam S, Gui J (2016) Treatment for Rheumatoid Arthritis and Risk of Alzheimer's Disease: A Nested Case-Control Analysis. CNS Drugs 30: 1111-1120.

22. Kao LT, Kang JH, Lin HC, Huang CC, Lee HC, et al. (2016) Rheumatoid Arthritis Was Negatively Associated with Alzheimer's Disease: A Population-Based Case-Control Study. PLoS One 11: e0168106.

23. Policicchio S, Ahmad AN, Powell JF, Proitsi P (2017) Rheumatoid arthritis and risk for Alzheimer's disease: a systematic review and meta-analysis and a Mendelian Randomization study. Sci Rep 7: 12861.

24. Ferraccioli G, Carbonella A, Gremese E, Alivernini S (2012) Rheumatoid arthritis and Alzheimer's disease: genetic and epigenetic links in inflammatory regulation. Discov Med 14: 379-388.

25. Sağ S, Sağ MS, Tekeoğlu I, Kamanlı A, Nas K, et al. (2017) Central nervous system involvement in rheumatoid arthritis: possible role of chronic inflammation and TNF blocker therapy. Acta Neurol Belg 124: 1-7.

26. Paouri E, Tzara O, Kartalou GI, Zenelak S, Georgopoulos S (2017) Peripheral Tumor Necrosis Factor-Alpha (TNF- $\alpha$ ) Modulates Amyloid Pathology by Regulating Blood-Derived Immune Cells and Glial Response in the Brain of AD/TNF Transgenic Mice. J Neurosci 37: 5155-5171.

27. Mamelak M (2017) Energy and the Alzheimer brain. Neurosci Biobehav Rev 75: 297-313.

28. Yin F, Sancheti H, Patil I, Cadenas E (2016) Energy Metabolism and Inflammation in Brain Aging and Alzheimer's Disease. Free Radic Biol Med 100: 108-122.

29. Daulatzai MA (2017) Cerebral hypoperfusion and glucose hypometabolism: Key pathophysiological modulators promote neurodegeneration, cognitive impairment, and Alzheimer's disease. J Neurosci Res 95: 943-972.

30. Rijpma A, van der Graaf M, Meulenbroek O, Olde Rikkert MGM, Heerschap A (2018) Altered brain high-energy phosphate metabolism in mild Alzheimer's disease: A 3-dimensional 31P MR spectroscopic imaging study. Neuroimage Clin 18: 254-261.

31. Orsucci D, Mancuso M, lenco EC, Simoncini C, Siciliano G, et al. (2013) Vascular factors and mitochondrial dysfunction: a central role in the pathogenesis of Alzheimer's disease. Curr Neurovasc Res 10: 76-80.

32. Safouris A, Hambye AS, Sculier C, Papageorgiou SG, Vasdekis SN, et al. (2015) Chronic brain hypoperfusion due to multi-vessel extracranial atherosclerotic disease: a potentially reversible cause of cognitive impairment. $J$ Alzheimers Dis 43: 23-27.
33. Pant S, Deshmukh A, Gurumurthy GS, Pothineni NV, Watts TE, et al. (2014) Inflammation and atherosclerosis-revisited. J Cardiovasc Pharmacol Ther 19: 170-178.

34. Viola J, Soehnlein O (2015) Atherosclerosis-A matter of unresolved inflammation. Semin Immunol 27: 184-193.

35. Tsounis D, Bouras G, Giannopoulos G, Papadimitriou C, Alexopoulos D, et al. (2014) Inflammation markers in essential hypertension. Med Chem 10: 672-681.

36. Pardo M, Porat Z, Rudich A, Schauer JJ, Rudich Y (2015) Repeated exposures to roadside particulate matter extracts suppresses pulmonary defense mechanisms, resulting in lipid and protein oxidative damage. Environ Pollut 210: 227-237.

37. Pardo M, Shafer MM, Rudich A, Schauer JJ, Rudich Y (2015) Single Exposure to near Roadway Particulate Matter Leads to Confined Inflammatory and Defense Responses: Possible Role of Metals. Environ Sci Technol 49: 8777-8785.

38. Carbone I, Lazzarotto T, lanni M, Porcellini E, Forti P, et al. (2014) Herpes virus in Alzheimer's disease: relation to progression of the disease. Neurobiol Aging 35: 122-129.

39. Kim GH, Kim JE, Rhie SJ, Yoon S (2015) The Role of Oxidative Stress in Neurodegenerative Diseases. Exp Neurobiol 24: 325-340.

40. Sweeney MD, Sagare AP, Zlokovic BV (2018) Bloodbrain barrier breakdown in Alzheimer disease and other neurodegenerative disorders. Nat Rev Neurol 14: 133-150.

41. Sahoo AK, Dandapat J, Dash UC, Kanhar S (2018) Features and outcomes of drugs for combination therapy as multi-targets strategy to combat Alzheimer's disease. $\mathrm{J}$ Ethnopharmacol 215: 42-73.

42. Howes MJ, Houghton PJ (2012) Ethnobotanical treatment strategies against Alzheimer's disease. Curr Alzheimer Res 9: 67-85.

43. Yang Y, Jia X, Feng J, Wang Z, Cao Y, et al. (2017) Fuzheng Quxie Decoction Ameliorates Learning and Memory Impairment in SAMP8 Mice by Decreasing Tau Hyperphosphorylation. Evid Based Complement Alternat Med 2017: 5934254.

44. Ong WY, Wu YJ, Farooqui T, Farooqui AA (2018) Qi Fu Yin-a Ming Dynasty Prescription for the Treatment of Dementia. Mol Neurobiol 55: 7389-7400.

45. Sun LM, Zhu BJ, Cao HT, Zhang XY, Zhang QC, et al. (2017) Explore the effects of Huang-Lian-Jie-Du-Tang on Alzheimer's disease by UPLC-QTOF/MS-based plasma metabolomics study. J Pharm Biomed Anal 151: 75-83.

46. Hu Q, Yu B, Chen Q, Wang Y, Ling Y, et al. (2018) Effect of Linguizhugan decoction on neuro- inflammation and expression disorder of the amyloid $\beta$-related transporters RAGE and LRP-1 in a rat model of Alzheimer's disease. Mol Med Rep 17: 827-834.

47. Hou Y, Wang Y, Zhao J, Li X, Cui J, et al. (2014) Smart Soup, a traditional Chinese medicine formula, ameliorates amyloid pathology and related cognitive deficits. PLoS One 9: e111215. 